\title{
BREVE ANÁLISE DA OBRA THE DESCRIPTION AND USE OF THE DOUBLE HORIZONTALL DYALL (1632) DE WILLIAM OUGHTRED
}

\author{
BRIEF ANALYSIS OF THE WORK THE DESCRIPTION AND USE OF \\ THE DOUBLE HORIZONTAL DYALL (1632) BY WILLIAM OUGHTRED
}

\author{
Verusca Batista Alves ${ }^{1}$ \\ Instituto Federal de Educação, Ciência e Tecnologia - IFCE \\ Ana Carolina Costa Pereira ${ }^{2}$ \\ Universidade Estadual do Ceará - UECE
}

\begin{abstract}
Resumo
Dentre as formas de inserir a história da matemática nas aulas, o uso de documentos históricos é uma temática que chama a atenção da maioria dos professores. Porém, pode ser um trabalho de difícil realização quando não se sabe a melhor forma de inserir esses materiais nas aulas. Tudo parte inicialmente da própria dificuldade do professor em pesquisar e ter acesso a esse tipo de material. Dessa forma, este trabalho apresenta uma breve análise da obra The description and use of the double horizontall dyall de 1632, com o objetivo de aproximar o leitor a um documento que trata do uso de um instrumento antigo denominado sundial ou relógio de sol. Para atingir esse objetivo, esta pesquisa apresenta inicialmente um perfil bibliográfico. Em seguida, o estudo caracteriza-se como documental e descritivo, que visa à estrutura física e o conteúdo da obra, buscando expor e descrever as características abordadas. Com a análise inicial da obra, nota-se as diversas possibilidades relacionadas ao ensino, como também na pesquisa. Porém, trata-se de uma obra do século 17 e dificuldades surgiram como, a grafia do inglês da época e algumas falhas no texto que impossibilitaram a leitura total do livro. Com isso espera-se que o leitor possa conhecer brevemente o conteúdo presente na obra e que, com trabalhos desse tipo, se possa auxiliar o professor que deseja utilizar esse tipo de material no ensino dos conteúdos matemáticos, facilitando a conexão entre história da matemática e o ensino. Sabe-se que ainda é uma análise superficial e que, portanto, pretende-se dar continuidade na investigação do conteúdo a obra.
\end{abstract}

Palavras-chave: História da Matemática; Fontes Históricas; William Oughtred; Horizontall Dyall.

\begin{abstract}
Among the ways to insert the history of mathematics in the classes, the usage of historical documents is a theme that draws the attention of most teachers. However, it can be a difficult task to do when you do not know the best way to introduce these materials in the classes. Everything initially comes from the teacher's own difficulty in researching and accessing this type of material. Thus, this paper presents a brief analysis of the work The description and use of the double horizontall dyall from 1632, aiming
\end{abstract}

\footnotetext{
1 veruscah.alves@gmail.com

2 carolina.pereira@uece.br
} 
at bringing the reader closer to a document that deals with the use of an old instrument $\mathrm{n}$ amed sundial. In order to reach the goal, this research firstly presents a bibliographic profile. Then, the study is characterized as documentary and descriptive, which focus on the physical structure and the content of the book, seeking to expose and describe the characteristics approached. By the initial analysis of the work, we can notice various possibilities related to teaching as well as researching. Yet, it is a piece of work from the 17th century, so difficulties have arisen, such as the spelling of the English and some failures in the text that made it impossible to fully read the book. Therefore, it is expected that the reader be able to know briefly the content of the book and that papers of this kind be likely to assist the teacher who wishes to use this material in teaching mathematical contents, besides facilitating the connection between the history of mathematics and education. It is known that it is still a superficial analysis and, therefore, it is intended to continue the investigation of the content of the work.

Keywords: History of Mathematics; Historical Sources; William Oughtred; Horizontall Dyall.

\section{Introdução}

Pesquisas relacionadas ao ensino de Matemática vêm crescendo nos últimos anos. No Brasil, Fiorentini (1994) abordou em sua tese os estudos que vinham sendo desenvolvidos na área da Educação Matemática até então. Com isso, destacou precisamente que, somente a partir da década de 1990, quando a Educação Matemática passou a ser uma comunidade científica, que os estudos nessa área começaram a amadurecer (FIORENTINI, 1994). Dessa forma, nota-se que é uma área investigada de forma recente, mas que vem tomando seu espaço no meio acadêmico.

Após esse período, várias temáticas surgiram dentro das pesquisas em Educação Matemática. Considerados pelas autoras Alves e Pereira (2016) e Silva (2016) como tendências matemáticas, a Modelagem Matemática, Jogos Matemáticos, Etnomatemática, Leitura e Escrita em Matemática, Resolução de Problemas, História da Matemática, Tecnologias da Informação e Comunicação, entre outras, configuram-se como formas de minimizar as dificuldades no ensino dos conteúdos matemáticos, proporcionando assim uma aprendizagem com mais significância.

Como citado, a História da Matemática faz parte desse grupo e é pesquisada por diversos autores que buscam explorar as potencialidades e possibilidades, e até mesmo, os pontos que se contrapõem ao seu uso na sala de aula.

Em se tratando dos possíveis benefícios da história para o ensino, autores como D’Ambrósio (2007), Pereira (2015), Saito (2014) exploram as potencialidades pedagógicas da história da matemática de diversas formas: tais como, o uso de fontes históricas, os instrumentos matemáticos, e até mesmo a influência cultural. Porém, além 
dos benefícios da história no ensino, é necessário destacar um importante aspecto relacionado diretamente esse uso: a dificuldade dos professores de matemática.

No contexto escolar, muitos fatores influenciam na aprendizagem dos alunos, e a formação do professor certamente é um desses. Alguns relatam dificuldades quanto ao uso da história, como falta de tempo, falta de material, sendo tais situações de ordem diária, talvez, as principais motivações quanto ao receio do uso da história da matemática.

Nesse aspecto, buscando unir a história da matemática com o ensino, esse artigo adentra-se a um estudo específico com a temática de fontes históricas, apresentando uma breve análise da obra The description and use of the double horizontall dyall, de William Oughtred.

\section{Aspectos teóricos e Metodológicos}

A variação de meios didáticos para o Ensino de Matemática são temas bastante discutidos em pesquisas no âmbito acadêmico. Por meio dos estudos, constata-se a importância de aulas diversificadas, quanto a recursos e métodos, que vão além da exposição do conteúdo, principalmente quando se trata da Matemática.

Em se tratando da utilização da história da matemática, é essencial que o professor conheça e saiba como aproveitar-se do que lhe é disponível para proporcionar a aprendizagem dos seus alunos. No entanto, é do conhecimento geral que existem dificuldades relacionadas ao uso.

Parte desse problema é relativo ao próprio professor de matemática, que, por situações profissionais, questiona cenários em que, a falta de tempo, de material e de experiência, são os principais casos no qual o uso da história da matemática encontra-se barrado.

Dessa forma, buscando auxiliar o professor nesse processo, apresenta-se uma fonte histórica que relaciona potencialidades didáticas no ensino de conteúdos matemáticos. Assim, inicialmente, apresenta-se a definição de Xavier (2010, p. 1100), sobre fontes históricas:

todos os tipos de vestígios inscritos no passado como um livro de receita, fotografias, cinema, músicas, enfim uma série de elementos que auxiliariam o historiador na busca de compreender como se estabeleceram os homens do 
passado, qual significado tais objetos adquiram para estas sociedades, para os grupos que o forjaram e no que tange sua relação com o presente.

Silva (2013, p. 38) ainda complementa essa definição afirmando que as fontes são "vestígios do passado, deixados por sociedades a e agentes, que nos permitem desvendar seus hábitos, seus costumes, suas produções culturais e, principalmente, suas formas de organização". Dessa forma, ainda podemos citar autores como Jahnke (2002), Pereira e Pereira (2015), Esteve (2011), entre outros, que abordam o uso das fontes.

Autores como Oliveira (2010), Baroni, Texeira e Nobre (2004) Saito e Dias (2011) destacam que a história abrange diferentes saberes que permitem uma formação de conceito mais ampla. Dessa forma, o uso de fontes históricas no ensino permite que se conheça aspectos mais gerais sobre o conteúdo, tais como, sociais, políticos, econômicos, religiosos, entre outros.

A fonte que é tratada neste trabalho refere-se à obra de William Oughtred (15741660), um professor inglês conhecido por suas réguas de cálculo, e apresenta o uso do instrumento denominado sundial, ou relógio de sol, dispositivo que realizava medições referentes a tempo.

Para a realização desta pesquisa, inicialmente se fez uma pesquisa do tipo bibliográfica, que, segundo Marconi e Lakatos (2003) é importante, pois é dessa forma que é possível levantar dados recentes a respeito de publicações do mesmo tipo. Dessa forma, ao buscar-se sobre do tratamento da obra The description and use of the horizontall dyall, nada foi encontrado.

Nesse momento, também se buscou por possíveis traduções de documentos semelhantes da época, como forma de apoiar a nossa tradução da obra, que, embora esteja aqui sendo exposto somente os tópicos, é um trabalho que continua sendo desenvolvido com o objetivo de compreender todo o conteúdo do livro.

Como a investigação dá-se por meio da análise de uma obra, também é uma pesquisa do tipo documental que, "vale - se de materiais que não receberam ainda um tratamento analítico, ou que ainda podem ser reelaborados de acordo com os objetos da pesquisa (GIL, 1991 p. 28)”. Assim, seguindo essa definição, entende-se a relevância da pesquisa que está sendo desenvolvida.

Também, de acordo o objetivo de investigação, este artigo evidencia-se como uma pesquisa descritiva que busca "descrever ou caracterizar com detalhes uma situação, um fenômeno ou um problema (FIORENTINI; LORENZATO, 2006, p.70)”. 
Nesse caso, se escolheu iniciar essa descrição pelos tópicos presentes na obra, dando início ao tratamento do livro. Ressalta-se que posteriormente planeja-se continuar o estudo, fornecendo dados mais detalhados a respeito da obra, de maneira a complementar este trabalho, tanto em seu conteúdo, quanto em seu objetivo.

\section{Análise preliminar da obra}

Inicialmente, é possível notar informações referentes à sua época, como o idioma Inglês, com traços em Latim, como por exemplo, a grafia de determinadas palavras que originalmente nos remete a um "V" e que atualmente temos como "U". Outra característica possível de ser apreciada logo na contracapa da obra é a origem de sua impressão, em Londres, e seu ano, apresentado ainda em algarismos romanos MDCXXXI, ou, 1632.

Oughtred tinha um fascínio pela medição do tempo, "Por causa desse interesse literário, científico e prático nos métodos de indicar o tempo, não é surpreendente que Oughtred tenha se dedicado ao domínio e ao avanço dos métodos de medição do tempo (CAJORI, 1916, p. 52, tradução nossa) ${ }^{3}$. Cajori (1916) cita também que The description and use of the double horizontall dyall (Figura 01) foi anexada em 1653 à outra obra, Récréations Mathématiques (1624) de Henry van Etten, um pseudônimo o Francês Jean Leurechon (1591-1690).

\footnotetext{
${ }^{3}$ Because of this literary, scientific, and practical interest in methods of indicating time it is not surprising that Oughtred devoted himself to the mastery and the advancement of methods of time-measurement (CAJORI, 1916, p. 52)
} 


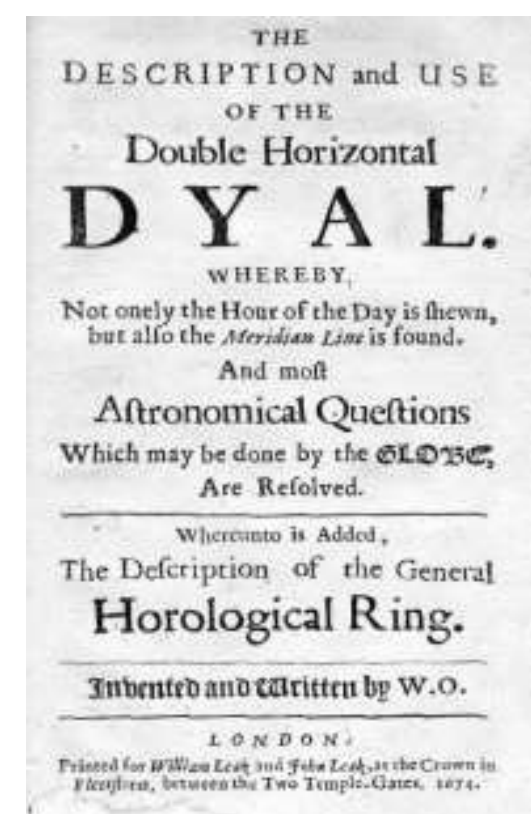

Figura 01 - Capa da obra The description and use of the double horizontall dyall, 1674 Fonte: Oughtred (1674, capa)

A obra que analisamos é de 1632, porém, devido ao desgaste da obra, buscamos pela capa da edição de 1674 para uma melhor visualização do leitor, sobre as informações aqui dadas. Esclarecemos que não tivemos acesso a obra de 1674 por completo, por isso, não pudemos aprimorar os dados aqui expostos por não termos material suficiente de comparação.

Outro aspecto que podemos destacar, é que a obra de 1632 apresenta algumas páginas de difícil visualização, no qual tivemos dificuldade de compreender o que estava escrito e dessa forma alguns termos não puderam ser totalmente interpretados.

Como o próprio título já mostra, a obra descreve o objeto e o uso da horizontall dyall ou sundial. Em suas páginas iniciais, Oughtred descreve o instrumento como um prato com dois diferentes discos. O disco mais externo é dividido em horas e quarters, que entendemos como quarto de hora, ou quadrante do círculo. Cada quadrante ou quarto de hora é dividido em três partes de 5', totalizando a hora toda como 60'.

O outro disco, interno, é a projeção do hemisfério superior sobre a planície do horizonte. Nesse caso, autor explica que o horizonte está dividido em ambos os lados dos pontos de leste e oeste em graus, descrevendo como conhecemos atualmente. E o centro do instrumento é o Zenith, ou ponto vertical. A obra tem características de um manual de uso e não contém uma imagem do instrumento. 
O livro está dividido tópicos listados, com os possíveis usos do instrumento como mostra o Quadro 1. O item I não é visível no texto, portanto, listamos do segundo em diante.

Quadro 1 - Tópicos do livro The description and use of the double horizontall dyall (1632)

\begin{tabular}{|c|c|c|}
\hline Da obra & Tradução nossa & Descrição \\
\hline $\begin{array}{l}\text { II. ufe. To find the } \\
\text { declination of the funne } \\
\text { every day }\end{array}$ & $\begin{array}{l}\text { II. Uso. Para encontrar a } \\
\text { declinação do sol (?) todos os dias }\end{array}$ & $\begin{array}{l}\text { Contém uma explicação } \\
\text { e } 3 \text { (três) exemplos }\end{array}$ \\
\hline $\begin{array}{l}\text { III. Vfe. To find the diurnall } \\
\text { arch, or circle of the funnes } \\
\text { courfe every day }\end{array}$ & $\begin{array}{l}\text { III. Uso. Para encontrar o arco } \\
\text { diurno, ou circulo do cuso do sol } \\
\text { (?) todos os dias }\end{array}$ & $\begin{array}{l}\text { Contém uma explicação } \\
\text { e } 3 \text { (três) exemplos }\end{array}$ \\
\hline $\begin{array}{l}\text { IIII. Vfe. To find the rifing } \\
\text { and fetting of the funne } \\
\text { every day }\end{array}$ & $\begin{array}{l}\text { IIII. Uso. Para encontrar o nascer } \\
\text { (?) e pôr(?) do sol (?) todos os dias }\end{array}$ & $\begin{array}{l}\text { Contém a explicação de } \\
\text { como usar }\end{array}$ \\
\hline $\begin{array}{l}\text { V. ufe. To know the reafon } \\
\text { and manner of the } \\
\text { Increafing and decreafing of } \\
\text { the dayes \& nights } \\
\text { throughout the whole yeare }\end{array}$ & $\begin{array}{l}\text { V. Uso. Para conhecer a razão e a } \\
\text { maneira do aumento e da } \\
\text { diminuição dos dias e noites } \\
\text { durante todo o ano }\end{array}$ & $\begin{array}{l}\text { Contém a explicação de } \\
\text { como usar }\end{array}$ \\
\hline $\begin{array}{l}\text { VI. Vfe. To find how farre } \\
\text { the funne rifeth, and fetteth } \\
\text { from the true Eaft and weft } \\
\text { points, which is called the } \\
\text { funnes: (não é possível ler o } \\
\text { restante do item) }\end{array}$ & $\begin{array}{l}\text { VI. Uso. Para descobrir quão longe } \\
\text { (?) o sol (?) se levanta, e se separa } \\
\text { dos pontos verdadeiros do Leste e } \\
\text { Oeste, que é chamado: (não é } \\
\text { possível ler o restante do item) }\end{array}$ & $\begin{array}{l}\text { Contém a explicação de } \\
\text { como usar }\end{array}$ \\
\hline $\begin{array}{l}\text { VII. Vfe. To finde the } \\
\text { length of every day and } \\
\text { night }\end{array}$ & $\begin{array}{l}\text { VII. Uso. Para encontrar o } \\
\text { comprimento de cada dia e noite }\end{array}$ & $\begin{array}{l}\text { Contém a explicação de } \\
\text { como usar }\end{array}$ \\
\hline $\begin{array}{l}\text { VIII. Vfe. To find the true } \\
\text { place of the funne upon the } \\
\text { dyall, that is the point of the } \\
\text { inftrument which anfwerth } \\
\text { to the place of the funne in } \\
\text { the heavens at any time, } \\
\text { which is the very ground of } \\
\text { all the queftions following }\end{array}$ & $\begin{array}{l}\text { VIII. Uso. Para encontrar o } \\
\text { verdadeiro lugar do sol sobre o } \\
\text { disco, esse é o ponto do } \\
\text { instrumento que atende ao lugar do } \\
\text { sol nos céus a qualquer momento, } \\
\text { que é o fundamento de todas as } \\
\text { questões seguintes }\end{array}$ & $\begin{array}{l}\text { Contém a explicação de } \\
\text { como usar }\end{array}$ \\
\hline $\begin{array}{l}\text { IX. Vfe. To find the hower } \\
\text { of the day }\end{array}$ & $\begin{array}{l}\text { IX. Uso. Para encontrar a hora (?) } \\
\text { do dia }\end{array}$ & $\begin{array}{l}\text { Contém a explicação de } \\
\text { como usar }\end{array}$ \\
\hline $\begin{array}{l}\text { X. Vfe. To find out the } \\
\text { Meridian, and other points } \\
\text { of the compaffe }\end{array}$ & $\begin{array}{l}\text { X. Uso. } \\
\text { Meridiano e e outros pontos da } \\
\text { bússola }\end{array}$ & $\begin{array}{l}\text { Contém a explicação de } \\
\text { como usar }\end{array}$ \\
\hline nd out the & Para descobrir o Azumith & Con \\
\hline
\end{tabular}




\begin{tabular}{|c|c|c|}
\hline $\begin{array}{l}\text { Azumith of the funne, that is } \\
\text { the diftance of the Verticall } \\
\text { circle, in which the funne is } \\
\text { at that prefent, from the } \\
\text { Meridian }\end{array}$ & $\begin{array}{l}\text { do sol, essa é à distância do círculo } \\
\text { vertical, em que o sol está } \\
\text { presente, do Meridiano }\end{array}$ & como usar \\
\hline $\begin{array}{l}\text { XII. Vfe. To find out the } \\
\text { Declination of any Wall } \\
\text { upon which the funne } \\
\text { fhineth, that is, how farre } \\
\text { that wall fwerveth from the } \\
\text { North or South, either } \\
\text { Eaftward or Weftward. }\end{array}$ & $\begin{array}{l}\text { XII. Usar. Para descobrir a } \\
\text { Declínio de qualquer Muralha } \\
\text { sobre a qual o sol brilha, ou seja, } \\
\text { até que ponto essa parede se } \\
\text { desvia do Norte ou do Sul, tanto } \\
\text { para o Oriente como para o } \\
\text { Ocidente }\end{array}$ & $\begin{array}{l}\text { Contém a explicação de } \\
\text { como usar }\end{array}$ \\
\hline $\begin{array}{l}\text { XIII. Vfe. How to place the } \\
\text { dyall upon a poft without } \\
\text { any other direEtion but it } \\
\text { felfe }\end{array}$ & $\begin{array}{l}\text { XIII. Uso. Como colocar o disco } \\
\text { em uma disposição sem qualquer } \\
\text { outra direção, mas ele próprio }\end{array}$ & $\begin{array}{l}\text { Contém a explicação de } \\
\text { como usar }\end{array}$ \\
\hline $\begin{array}{l}\text { XIIII. Vfe.To find the height } \\
\text { of the Sun at high noone } \\
\text { every day }\end{array}$ & $\begin{array}{l}\text { XIIII. Uso. Para encontrar a altura } \\
\text { do Sol meio dia todos os dias }\end{array}$ & $\begin{array}{l}\text { Contém a explicação de } \\
\text { como usar }\end{array}$ \\
\hline $\begin{array}{l}\mathrm{XV} \text {. Vfe. To find the height } \\
\text { of the funne at any hower or } \\
\text { time of the day }\end{array}$ & $\begin{array}{l}\text { XV. Uso. Para encontrar a altura } \\
\text { do sol (?) a qualquer hora (?) ou } \\
\text { momento (?) do dia }\end{array}$ & $\begin{array}{l}\text { Contém a explicação de } \\
\text { como usar }\end{array}$ \\
\hline $\begin{array}{l}\text { XVI. Vfe. The height of the } \\
\text { funne being given to finde } \\
\text { out the hower, or what it is a } \\
\text { clocke }\end{array}$ & $\begin{array}{l}\text { XVI. Uso. A altura do sol (?) que } \\
\text { está sendo dada para descobrir a } \\
\text { hora, ou o que é um relógio }\end{array}$ & $\begin{array}{l}\text { Contém a explicação de } \\
\text { como usar }\end{array}$ \\
\hline $\begin{array}{l}\text { XVII. Vfe. Confiderations } \\
\text { for the ufe of the inftrument } \\
\text { in the night }\end{array}$ & $\begin{array}{l}\text { XVII. Uso. Considerações sobre o } \\
\text { uso do instrumento durante a noite }\end{array}$ & $\begin{array}{l}\text { Contém a explicação de } \\
\text { como usar }\end{array}$ \\
\hline $\begin{array}{l}\text { XVIII. Vfe. To find how } \\
\text { many degrees the funne is } \\
\text { under the horizon at any } \\
\text { time of the night }\end{array}$ & $\begin{array}{l}\text { XVIII. Uso. Para descobrir a } \\
\text { quantos graus o sol está sob o } \\
\text { horizonte a qualquer hora da noite }\end{array}$ & $\begin{array}{l}\text { Contém a explicação de } \\
\text { como usar }\end{array}$ \\
\hline $\begin{array}{l}\text { XIX. Vfe. To find out the } \\
\text { length of the Crepufculum, } \\
\text { or twylight, every day }\end{array}$ & $\begin{array}{l}\text { XIX. Uso. Para descobrir o } \\
\text { comprimento do Crepusculum, ou } \\
\text { crepúsculo, todos os dias }\end{array}$ & $\begin{array}{l}\text { Contém a explicação de } \\
\text { como usar }\end{array}$ \\
\hline $\begin{array}{l}\text { XX. Vfe. If the day of the } \\
\text { month be not knowne, to } \\
\text { find it out by the dyall }\end{array}$ & $\begin{array}{l}\text { XX. Uso. Se o dia do mês não for } \\
\text { conhecido, procure-o pelo disco }\end{array}$ & $\begin{array}{l}\text { Contém a explicação de } \\
\text { como usar }\end{array}$ \\
\hline
\end{tabular}

Fonte: Elaborado pelas autoras

Como é possível notar, o idioma é do século XVII e possui grafias e palavras que, com o tempo, mudaram. Por isso, ao fazermos a tradução da obra, buscamos compreender o máximo possível da ideia do autor, para que não houvesse perca de nenhuma informação importante. 
Assim, traduzimos algumas palavras de forma que o contexto da frase pudesse fazer sentido, mas deixamos a especificação ao lado, para que o leitor compreendesse que pode ser algo mutável. Também, preferimos não fazer adaptações na grafia para traduzir o texto e dessa forma preservar a ideia original.

Nossa intenção ao traduzir inicialmente os tópicos, é apresentar de forma concisa o conteúdo presente na obra, para que assim possa servir de auxílio para pesquisadores que desejam investigar essa obra.

\section{Algumas considerações finais}

O uso de fontes históricas no ensino é um recurso da história da matemática que permite ao aluno conhecer diversos aspectos relacionados ao assunto matemático que está sendo estudado. Por isso, de forma breve, este trabalho quis expor alguns detalhes da obra The description and use of the double horizontall dyall, de forma a auxiliar professores que buscam por fontes históricas para utilizarem nas aulas de matemática.

Ao realizar esse estudo, foi possível notar dificuldades inerentes a esse tipo de trabalho, como, a leitura de um idioma do século XVII e as condições do texto que não permitiram a total apreciação do conteúdo.

Nota-se que o estilo da escrita nos remete a um manual que ensina os possíveis usos do instrumento que está relacionado à obra, e que, mesmo sendo um relógio de sol, era também manuseado no período noturno.

Portanto, diante dessas características, esta obra abre diversas possibilidades quanto a sua utilização em sala de aula, porém, sabe-se que é uma análise ainda superficial, em que, também se abre espaço para futuras pesquisas acadêmicas a respeito.

Por isso, posteriormente buscaremos trabalhar mais profundamente a obra, analisando o texto por completo. Por hora, deixamos essas informações iniciais, pois se trata de um trabalho que está em constituição.

\section{Referências}

ALVES, Verusca Batista; PEREIRA, Ana Carolina Costa. Instrumentos históricos e o ensino de Matemática: a Régua de Cálculo Circular e suas contribuições na formação do professor. Remat, Bento Gonçalves, v. 2, n. 2, p.39-50, nov. 2016. 
BARONI, Rosa Lúcia Sverzut; TEIXEIRA, Marcos Vieira; NOBRE, Sergio Roberto. A investigação científica em História da Matemática e suas relações com o programa de pósgraduação em Educação Matemática. In: BICUDO, Maria Aparecida Viggiani; BORBA, Marcelo de Carvalho. Educação Matemática: pesquisa em movimento. São Paulo: Cortez, 2004. p. 164-185.

CAJORI, Florian. William Oughtred: a great seventeenth-century teacher of mathematics. Chicago: The Open Court Publishing Company, 1916.

D'AMBROSIO, Beatriz Silva. Reflexões sobre a História da Matemática na formação de professores. Revista Brasileira de História da Matemática, Especial, n. 1, p. 399406, dez. 2007.

ESTEVE, Maria Rosa Massa et al. Understanding Mathematics using original sources. Criteria and Conditions. In: BARBIN, Evelyne; KRONFELLNER, Manfred, TZANAKIS, Constantinos. (Eds.). History and Epistemology in Mathematics Education. Proceedings of the Sixth European Summer University. Vienna: Verlag Holzhausem GmbH, 2011, p. 415 - 428.

FIORENTINI, Dario. Rumos da pesquisa brasileira em educação matemática: o caso da produção científica em cursos de pós-graduação. 1994. 414 f. Tese (Doutorado) - Curso de Educação, Faculdade de Educação, Universidade Estadual de Campinas, Campinas, 1994.

FIORENTINI, Dario; LORENZATO, Sergio. Investigação em educação matemática: percursos teóricos e metodológicos. Campinas: Autores Associados, 2006.

GIL, Antonio Carlos. Como elaborar projetos de pesquisa. São Paulo: Editora Atlas, 1991.

JAHNKE, Hans Niels. The use of original sources in the mathematics classroom. In: FAUVEL, John; VAN MAANEN, Jan. History in Mathematics Education. New York, Boston, Dordrecht, London, Moscow: Kluwer Academic Publishers, 2002. Cap. 9. p. 291-328.

MARCONI, Marina de Andrade; LAKATOS, Eva Maria. Fundamentos de Metodologia Científica. 5. ed. São Paulo: Atlas, 2003.

OLIVEIRA, Rosalba Lopes. Artefatos históricos: mediando saberes na formação docente do professor que ensina Matemática. In: ENCONTRO NACIONAL DE EDUCAÇÃO MATEMÁTICA, 10., 2010, Salvador, BA. Anais ... Salvador, BA: SBHmat, 2010.

OUGHTRED, William. The description and use of the double horizontall dyall (1632). Londres: Eebo, 1632. William Forster.

PEREIRA, Ana Carolina Costa. Aspectos históricos da régua de cálculo para a construção de conceitos matemáticos. São Paulo: Livraria da Física, 2015. 
PEREIRA, Ana Carolina Costa; PEREIRA, Daniele Esteves. Ensaio sobre o uso de fontes históricas no ensino de Matemática. REMATEC. Revista de Matemática, Ensino e Cultura (UFRN), v. 10, p. 65-78, 2015.

SAITO, Fumikazu. Instrumentos matemáticos dos séculos XVI e XVII na articulação entre história, ensino e aprendizagem de matemática. REMATEC, v. 9, n. 16, p. 25-47, mai./ago. 2014.

SAITO, Fumikazu; DIAS, Marisa da Silva. Articulação de entes matemáticos na construção e utilização de instrumentos de medida do século XVI. Natal: Sociedade Brasileira de História da Matemática, 2011.

SILVA, Ana Paula Pereira do Nascimento. A leitura de fontes antigas e a formação de um corpo interdisciplinar de conhecimentos: Um exemplo a partir do Almagesto de Ptolomeu. 2013. 100 f. Dissertação (Mestrado em Ensino de Ciências Naturais e Matemática) - Universidade Federal do Rio Grande do Norte, Natal, 2013.

SILVA, Isabelle Coelho da. O uso de fontes na disciplina de História da Matemática a partir do problema 56 do Papiro de Rhind. 2016. 67 f. Trabalho de Conclusão de Curso (Graduação) - Universidade Estadual do Ceará, Fortaleza, 2016.

XAVIER, Erica da Silva. O uso das fontes históricas como ferramentas na produção de conhecimento histórico: a canção como mediador. Antíteses, vol. 3, n. 6, jul.-dez. de 2010, pp. 1097-1112. 